\title{
Investigation of Membrane Pinhole Effects in Polymer Electrolyte Fuel Cells by Locally Resolved Current Density
}

\author{
R. Lin, ${ }^{\text {a,b }}$ E. Gülzow, ${ }^{a}$ M. Schulze, ${ }^{a}$ and K. A. Friedrich ${ }^{\mathrm{a}, *, \mathrm{z}}$ \\ ${ }^{a}$ Institute of Technical Thermodynamics, German Aerospace Center, Stuttgart, D-70569 Germany \\ ${ }^{b}$ Clean Energy Automotive Engineering Center and School of Automotive Studies, Tongji University, \\ Shanghai, 201804 China
}

\begin{abstract}
In order to increase the reliability of fuel cells, an online diagnostic method for detection of operation malfunctions, as well as the early detection of failures in the fuel cells, is necessary. For this purpose, locally resolved current density measurements are an important tool, but the interpretation of the data related to the detection of malfunctions or failures is not straightforward. Here, segmented cell technology is applied to investigate the current density distributions in the anode and cathode electrodes to ascertain their equivalence due to the strong perpendicular coupling of currents. Current density distributions are further used to determine the signature of pinhole formation in the membrane. Different behavior is observed for membrane leakage under open circuit and under applied load conditions. Whereas the cell at open circuit is characterized by a positive current in the vicinity of the pinhole and small negative currents in the remaining area, an applied load leads to large negative currents at the pinhole. The characteristic behavior can be explained by high crossover rates of hydrogen from the anode to the cathode. The nongeneric signature is used to detect the deterioration of a membrane electrode assembly after a test stand malfunction. A sudden pressure drop associated with vaporation of water and the fast cooling of the cell is assumed to trigger the failure of the membrane. After $48 \mathrm{~h}$, fissures in several positions of the membrane near the edges of the cell holder are observed. Through the evolution of leakages in the fuel cell, a malfunction can be detected at an early stage and thereby catastrophic failure of the whole stack may be avoided or anticipated.

(C) 2010 The Electrochemical Society. [DOI: 10.1149/1.3504255] All rights reserved.
\end{abstract}

Manuscript submitted March 8, 2010; revised manuscript received September 25, 2010. Published November 9, 2010.

The durability of membranes has been improved dramatically during the last decade by chemical stabilization routines. These procedures consist of eliminating the carboxylic acid groups which are responsible for an autocatalytic radical attack. Radical formation has been reported to lead to thinning of membranes and eventually to pinhole formation. The degradation of membranes has been extensively studied. ${ }^{1-17}$ Even for chemical stabilized membranes the appearance of leakages is still an important issue as the mechanical strain during dry-wet cycling and the associated dimensional changes may lead to fissures and holes in membranes.

Weber has theoretically investigated membrane thinning and the formation of pinholes by sophisticated modeling. ${ }^{18}$ In this study, the changes in the performance and homogeneity of the currents are investigated as permeation of the various gases through the membrane increases. In a first case, operation at $120^{\circ} \mathrm{C}$ is simulated with a membrane of $25 \mu \mathrm{m}$ thickness with a fixed conductivity of $0.1 \mathrm{~S} / \mathrm{cm}$. Increasing gas-permeation rates lead to lowering performance especially in the low current density regime of the U(i) curve [a maximum limit for the hydrogen permeation coefficient of 1 $\times 10^{-10} \mathrm{~mol} /($ bar $\mathrm{cm} \mathrm{s})$ without affecting performance is determined]. At higher values the cell performance and current efficiency decrease greatly, leading to current reversal (negative currents) due to mixed potential at the anode and cathode. The negative currents can reach very high values, being locally much larger than the cell current density. The second case studied by Weber is the presence of a single pinhole or multiple pinholes in the membrane. The model also predicts negative current densities as well as temperature spikes if the pinhole area is larger than $0.1 \%$ of the active area. Multiple pinholes have a stronger detrimental effect depending on their specific locations. The work clearly demonstrates the influence of gas crossover (mainly hydrogen), which impacts performance and water as well as thermal management.

In order to achieve a better understanding of the electrochemical processes in polymer electrolyte membrane fuel cells (PEMFC) and to investigate effects of operating conditions on the local perfor-

* Electrochemical Society Active member.

z E-mail: andreas.friedrich@dlr.de mance and even the error detection of the fuel cell, a variety of locally resolved current density distribution mapping techniques have been developed. ${ }^{19-25}$

Achieving a homogeneous current density profile under a variety of operating conditions is still a major task in the development of fuel cells, which requires locally resolved in situ information. Heterogeneity in the current distribution can be a result of changes in the reactant concentration as well as of other influences, such as nonuniform contact pressure, temperature distribution, and water accumulation in some areas of the flow field. Efforts had been made to measure the current density distributions by application of the printed circuit board (PCB) technique to improve the accuracy of the experiment. ${ }^{26,27}$ At DLR, PCBs with segments and internal measurement resistances had been developed and used to study locally resolved current densities in various types of fuel cells or stacks. It is clear that PCB technology exhibits not only space advantages enabling the integration of segmented bipolar plates in very different geometries. Furthermore, segmented cells provide local properties of the fuel cell in addition to the integral performance in order to understand the degradation or failure of the fuel cells. ${ }^{27}$

Current density distributions can be influenced by both anode and cathode heterogeneities in the fuel cells, which is difficult to identify. Furthermore, the printed circuit board itself introduces a disturbance by additional resistances in the electrical circuit and also by changing the water interactions compared to the normal bipolar plate. The resulting complexity of the experimental setup has led to some uncertainties in the interpretation of results. ${ }^{22,28}$ It is commonly believed that current densities of both electrodes are identical because they are strongly coupled through the membrane conductivity. This depends, however, on the ratio of in-plane to out-of-plane electronic conductivity of electrodes, gas diffusion layers, and bipolar plates (or PCBs). Since the interpretation of the measurements here is based on the anode-sided PCB location, we wanted to ascertain the assumption of identical current density distributions independent of the segmented bipolar plate location. For that purpose, segmented bipolar plates are integrated on both sides of the cell. Single segmented arrangements are consecutively used to investigate pinhole failures of the membrane and to determine their signatures in the current density distributions. This study aims at early detection of membrane thinning to avoid the resulting catastrophic failure of the cells and the whole stack by adapting operation conditions. 


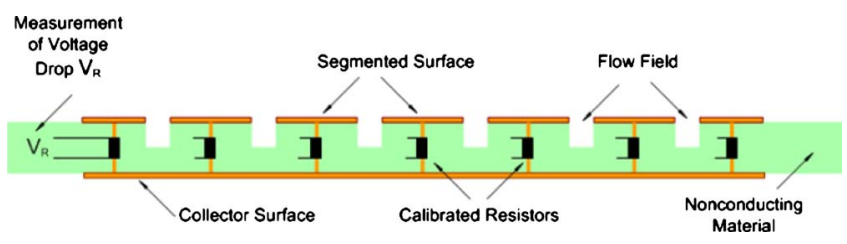

Figure 1. (Color online) Schematic diagram of the measuring unit (PCB) inside the fuel cell for current density distribution measurements.

\section{Experimental}

Principle of segmented bipolar plate.- As mentioned, the PCB technique allows a flexible design of shape and size of the current collector segments, which are placed on the surface of an epoxyglass resin matrix. The resistor array for the current measurement is integrated in the PCB using a multilayer assembly. The back side of the measuring plate is connected with the gas supply and bracing plate. The top and back layers of the board are connected with each other by conducting wires and the layer which is in contact with the Membrane Electrolyte Assembly (MEA) (i.e., segmented surface) is divided into segments to avoid lateral (i.e., in-plane) conduction (Fig. 1).

This setup forces the current between the segmented surface and the collector surface (back layer) on discrete pathways, which connect every segment surface with the collector surface. The current collector segments of the measuring board are gold plated to decrease the contact resistance and to avoid corrosion. The sensing wires on the segmented bipolar plate are connected to a data acquisition unit consisting of a multiplexer and a digital multimeter. The measurement setup works independently from the electric load unit and the control system of the fuel cell. The shunt resistance in the board is $\mathrm{R}\left(80^{\circ} \mathrm{C}\right)=4.3 \mathrm{~m} \Omega(1 \pm 0.5 \%)$.

Design of segmented bipolar plates.- A segmented bipolar plate with 49 segments for the single cell was used in this experiment. The schematic of the flow field (serpentine flow field) in relation to the segment in the PCB is shown in Fig. 2. thirty-three flow field channels were machined directly into the plate. The width of the channel and the land are 1.1 and $0.8 \mathrm{~mm}$, respectively. G1 is the inlet of the coflow configuration, whereas A7 is the location of the flow outlet. Coflow for fuel and oxidant is used for all presented measurements. To detect the coupling properties of anode and cathode electrode current distributions, two PCBs with segmented current collectors and flow fields are integrated at both electrodes for the current density distribution measurements. The scheme of current density measurement configuration with double printed circuit boards is shown in Fig. 3. It consists of MEA, gas diffusion layers, segmented printed circuit boards, and current collectors at anode and cathode sides of the cell.

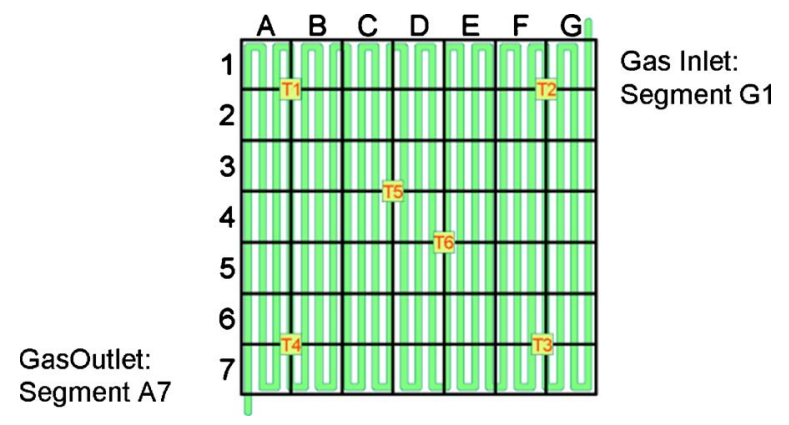

Figure 2. (Color online) Segmented flow field of current density distribution measurement board. T1-T6 are the temperature sensors for resistance calibration and temperature distribution measurements.

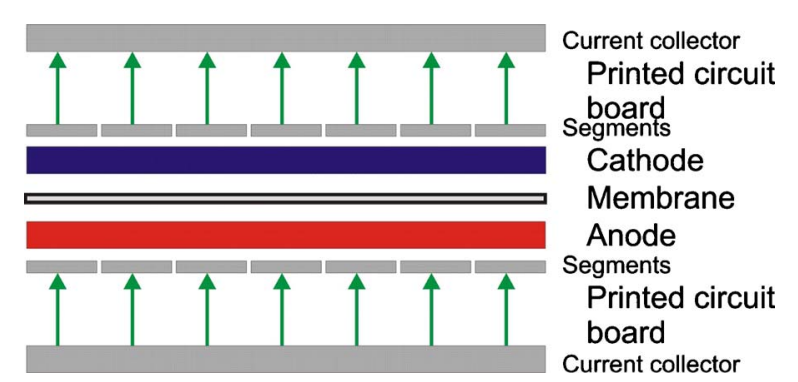

Figure 3. (Color online) Schematic diagram of current density measurement configuration with double printed circuit boards (the arrow points to the direction of the current).

Test procedure and operating conditions.- The MEA was obtained from Ion Power Company at an effective electrode area of $25 \mathrm{~cm}^{2}$ with a $25 \mu \mathrm{m}$ thick membrane. The gas diffusion layers from the SGL group (24/25BC) were placed on both sides of the MEA without hot-pressing steps. The cell was tested for gas leakage and crossover before operation in the test stand. Hydrogen stoichiometry $\left(\lambda \mathrm{H}_{2}\right)$ and air stoichiometry ( $\lambda$ air) were kept constant at 1.5 and 2 , respectively. The pressure of the single fuel cell is varied from 0.2 to $0.4 \mathrm{MPa}$, which is specified for each measurement. The operation temperature was either 80 or $120^{\circ} \mathrm{C}$, the latter for accelerated aging of the membrane.

Characterization of the fuel cell.- The morphological changes of membrane and catalyst were characterized by scanning electron microscopy (SEM) before and after operation. Images of the MEA were examined by a JSM-5600LV microscope to analyze and record the morphological changes of the electrodes and the connection between the catalyst layer and the membrane.

\section{Results and Discussion}

Coupling of anode and cathode current distributions. - In general, current may flow in three dimensions, and only in a special case of an anisotropic electronic conductor it would flow just in one direction. It is generally assumed that the local current density of both electrodes of the Polymer electrolyte membrane (PEM) fuel cell is identical due to their strong coupling but no direct evidence has been reported. An intact MEA is placed between two segmented bipolar plates in this experiment to compare the current distribution on both membranes and to evaluate the significance of lateral currents. Figure 4 displays the current density distributions measured in a galvanostatic mode at $80^{\circ} \mathrm{C}$ with both gas streams fully humidified. The global current is controlled at $9 \mathrm{~A}$ in this measurement, and variations of the current density are observed in different segments $\left(25 \mathrm{~cm}^{2}\right.$ active area; $\left.360 \mathrm{~mA} \mathrm{~cm}^{-2}\right)$. Even under normal operation conditions the current density distribution is heterogeneous over the active area with variations from 200 to $500 \mathrm{~mA} / \mathrm{cm}^{2}$ (Fig. 4). The higher values of the current density are located in the outlet part of the cell, while the lowest current density is found at the inlet region near G1, which normally indicates an insufficient humidification of the membrane in the inlet area.

In Fig. $4 \mathrm{a}$ and $\mathrm{b}$ the current density distribution at the anode and cathode are displayed. The heterogeneous distribution of the current densities appears to be very similar, both exhibiting the pronounced low current density area in the inlet region. In Fig. $4 \mathrm{c}$ the difference between both current density distributions is shown to expose the differences between both sides. The observed differences in Fig. 4c are quite small between +30 and $-10 \mathrm{~mA} / \mathrm{cm}^{2}$ and not associated with a specific area of high or low current density and indicate the accuracy limits of the measurement. It can be concluded that due to the still high conductivity perpendicular to the membrane plane (even though the shunt resistors are integrated) compared to the in-plane conductivity, the lateral currents are negligible and the anode and cathode current density distributions are equivalent. In ad- 

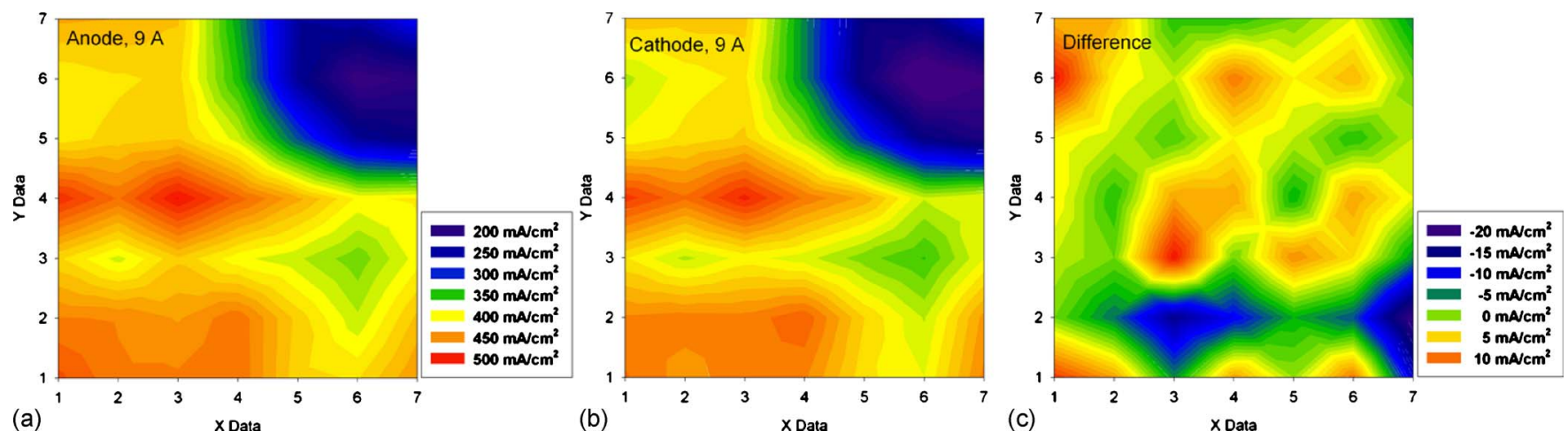

Figure 4. (Color online) Current density distributions measured at galvanostatic mode (9A) [an intact MEA with gases fully humidified at $80^{\circ} \mathrm{C}$ (cell and humidifier temperature)]. (a) Anode current density distribution; (b) cathode current density distribution, and (c) difference between anode and cathode current density distribution.

dition, the effect of the channel structure on the current density of segments is not evident in the measurement. However, it has to be stressed that the segment sizes are not small enough to resolve the finer channel structure.

For the following locally resolved measurements in this arrangement we can ascertain that a single segmented bipolar plate provides the accurate current density distribution of the whole cell. The lateral conductivity in the membrane has no significant influence as the membrane thickness is small compared to the segment size.

Pinhole detection by current density distribution.- Under "normal operation" of the cell-where the term "normal operation" means that the fuel cell has no leakages and that the fuel cell is operated without flooding of the cell, avoiding fuel starvation and other failure modes - the total (integral) current is zero for open circuit conditions independent of the operating parameter (gas flow, temperature, humidification, etc.). In this case, the gas concentrations and the other physical conditions in the cell are similar over the complete area and therefore the variations in the local potentials are very small and no significant local current density variation should exist. However, even at open circuit conditions, in some areas variations occur with different current directions; so in the normal operation, current density fluctuations of a few $\mathrm{mA} / \mathrm{cm}^{2}$ around zero are typical. ${ }^{29}$ Under load, the current density in each segment increases, leading concurrently to larger variations between segments. The variations in the fuel cell performance correspond mainly to local variations in the operating conditions at which the local humidification is of major importance.

With a leakage due to a pinhole in the membrane, the variations of the local operating conditions can dramatically increase. In particular, the composition of the reactants is changed strongly in the leakage area since the gases, in this case hydrogen and oxygen mix and react. Naturally, the heterogeneous reaction to water takes place on the catalyst surfaces in parallel to the electrochemical reactions. Consequently, at the leakage a local mixed potential will be formed as was also investigated by the paper of Weber. ${ }^{18}$

The mixed potentials are formed on the anode as well as on the cathode. However, the variation of the anode potential upon variation of the hydrogen concentration is smaller compared to the cathode potential variation upon changing the oxygen concentration. Furthermore, the oxygen reduction reaction has to be superimposed with the hydrogen oxidation reaction resulting from the permeated gas. The reason is that the reaction rates (determined by exchange current densities) at the anode are significantly higher compared to the reaction rates at the cathode (hydrogen oxidation exhibits exchange current densities in the order of $10 \mathrm{~mA} \mathrm{~cm}^{-2}$, whereas the oxygen reduction reaction possesses much lower exchange current densities in the order of $10^{-8} \mathrm{~A} \mathrm{~cm}^{-2}$ ). In addition, in the case of a leakage, typically more hydrogen crosses over compared to oxygen, especially if the cell is operated with air. The hydrogen diffusion coefficient is higher than for oxygen, and the oxygen concentration is diluted by nitrogen in air. Consequently, it can be generally expected that the variation of the local potential induced by the formation of the mixed potential is more significant at the cathode compared to the anode and this result is confirmed by Weber's simulations.

In Fig. 5 the current density distribution measured for a cell with a pinhole at $80^{\circ} \mathrm{C}$ which is formed accidentally under open circuit (Fig. 5a) and under load of ca. $100 \mathrm{~mA} \mathrm{~cm}^{-2}$ (Fig. 5b) is shown. The PCB used for the current density measurement is integrated only on the anode side of the fuel cell for all other measurements discussed here. The current density distributions in Fig. 5a and b show an unequal current density distribution which clearly marks the presence of a leakage associated with a pinhole. For open circuit conditions, in the area neighboring the leakage, a positive current is observed which corresponds to the fuel cell operation reaction mode. In contrast, the current in most of the residual area shows a current direction corresponding to a reverse current, meaning that the cell is operating in an electrolysis mode.

Due to the pinhole in the area, on the hydrogen electrode a reduction reaction and on the cathode an oxidation reaction take place. In the area directly influenced by the leakage a mixed potential is formed, leading to overpotentials on anode and cathode sides. This deviation from the equilibrium potential is not restricted just to the leakage area but influences nearby areas and drives the fuel cell reaction. In contrast, the distant areas are not influenced significantly by the leakages; therefore, the local potentials are deviating moderately in an opposite direction from the equilibrium potential and consequently the electrolysis reaction takes place in these areas. In this regard, the electrode can be considered a local-action cell which is driven by the continous gas crossover. Of course, the different electrochemical reactions at the open circuit condition compensate over the total electrode, but the mean open cell voltage is lower than in a cell with intact MEA.

The current distribution changes markedly under applied load conditions, as shown in Fig. 5b. The cell with a pinhole exhibits a larger heterogeneity of the current density distribution compared to an undamaged cell. In the current density measured under load the areas operating in the "fuel cell" mode and in the "electrolysis" mode are reversed compared to the current density observed at open circuit conditions. This is exactly the behavior reported by the simulation study of Weber for high gas permeation through the membrane or pinhole formation. From the discussed literature it is quite evident why the reversal of the current appears near the pinhole under load. This is illustrated in Fig. 6. This graph shows schematically the hydrogen and oxygen reactions on the reversible hydrogen potential scale. When hydrogen crosses to the cathode side (with higher rates compared to oxygen in air to the anode side) the hydrogen oxidation and oxygen reduction currents superimpose, leading 


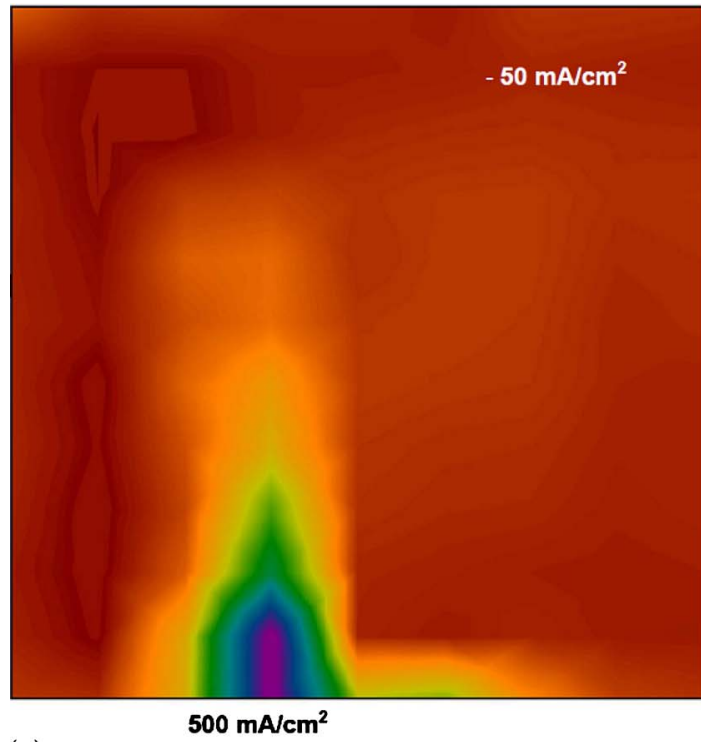

(a)
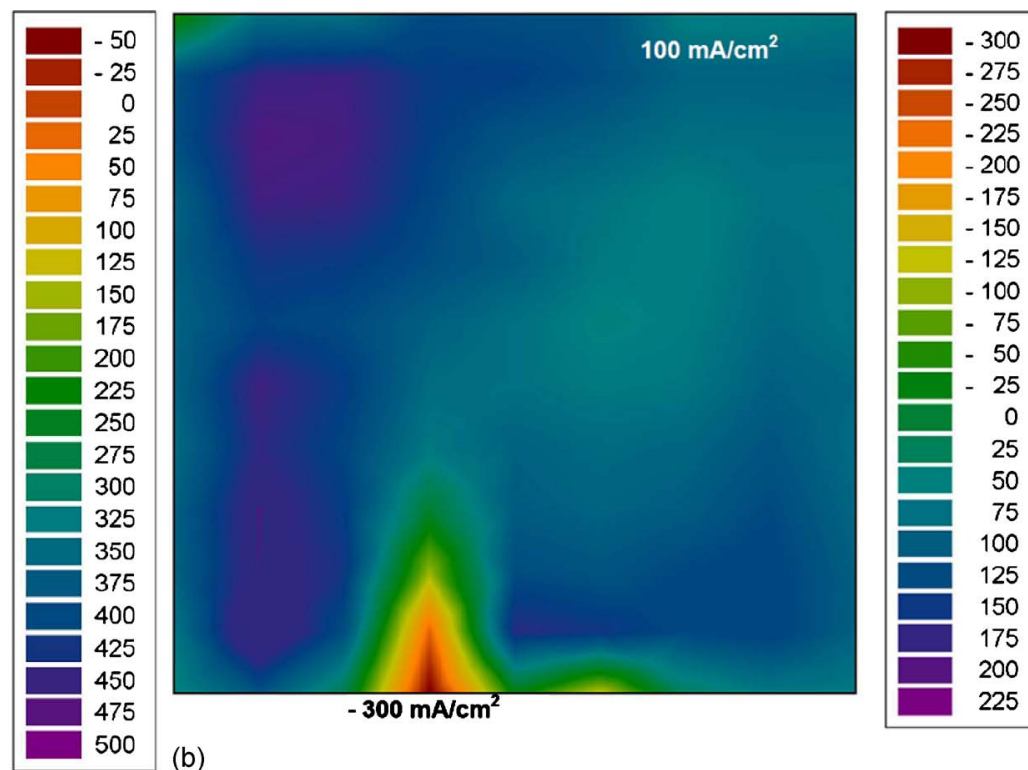

(b)

Figure 5. (Color online) Current density distribution measured on a MEA with a pinhole for (a) open circuit conditions and (b) under load (approx. $\left.100 \mathrm{~mA} / \mathrm{cm}^{2}\right)$.

to mixed potentials at the electrode. In a first approximation (neglecting loss of active area at the pinhole and water formation) the curve for the oxygen reaction can be shifted upward. If the hydrogen oxidation rate is higher compared to the original oxygen reduction rate, the current is revered, meaning that the positive electrode becomes the anode. To compensate, the negative electrode has to evolve hydrogen as a partner reaction and is now a local cathode. Due to the high exchange current density of the hydrogen reactions, the electrode potentials and the cell voltage do not change significantly under these conditions. Therefore, no major lateral currents which would equalize potential differences are expected.

The pronounced signature of a pinhole in the membrane can be used to follow the time evolution of a membrane leakage and to use current density distributions for malfunction detection, which is described in the following

Failure detection due to evolution of membrane leakage.- A different cell with the same manufacturing specifications was operated at $120^{\circ} \mathrm{C}$ at $0.4 \mathrm{MPa}$ (for both electrodes) and the humidification temperature of the anode and cathode was also set to $120^{\circ} \mathrm{C}$. These are highly accelerating degradation conditions which corre-

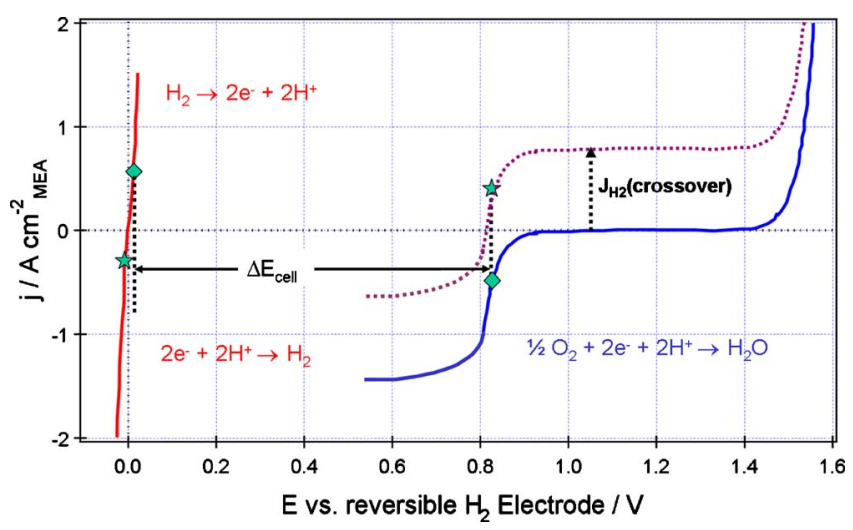

Figure 6. (Color online) Schematic representation of the influence of large cross-over rates of hydrogen on the reaction characteristics of the cell. When hydrogen oxidation at the cathodes exceed oxygen reduction current a current reversal is observed. spond to the temperature used in the theoretical investigations by Weber. The total durability of the cell under these conditions is found to be around $200 \mathrm{~h}$. A test stand related malfunction with an accidental pressure drop induced a membrane failure which was monitored and is described in detail.

Figure 7 monitors the evolution of global current and the cell temperature in the segmented cell area when the test stand malfunction happened. This incident is seen in the inset of Fig. 7 showing the time dependence of air and hydrogen pressure. It occurred 2 days before the membrane leakage started, which is described in Fig. 8 and 9. It is speculated that the sudden pressure drop of both gas streams initiated a membrane failure reported in the following. The temperature of the segments reflected precisely the real temperature of the MEA due to its close proximity to the membrane. Due to the test stand malfunction the air and hydrogen pressure rapidly decreased and at the same time the temperature decreased from 120 to $112^{\circ} \mathrm{C}$ in a relatively short time $(85 \mathrm{~s})$. Also, the global current diminished concurrently. The sudden drop of the hydrogen and air pressure causes evaporation of the liquid water present under this high humidity conditions and results in a large variation of local water content. The drop in pressure also induced a fast cooling of

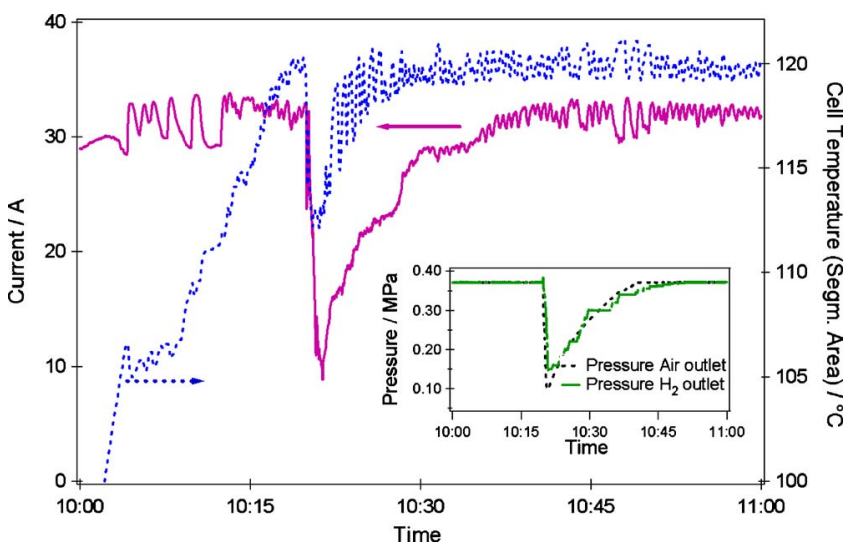

Figure 7. (Color online) Evolution of the current, the cell temperature and the pressures of hydrogen and air (in the insert), two days before the membrane leakage started. 

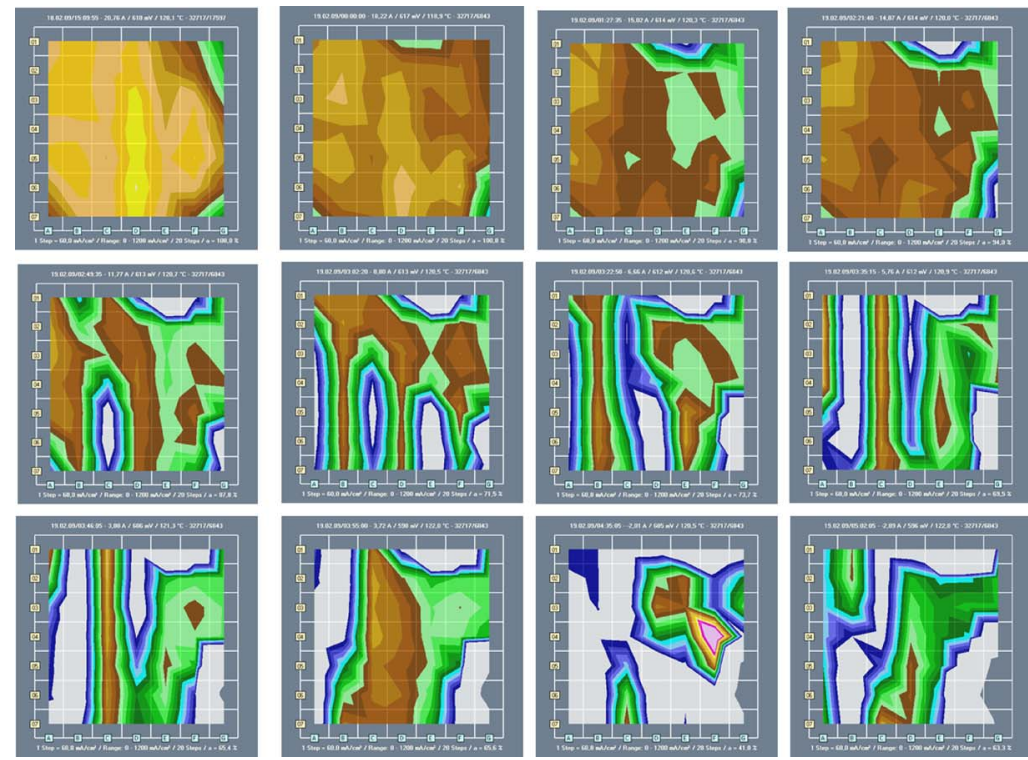

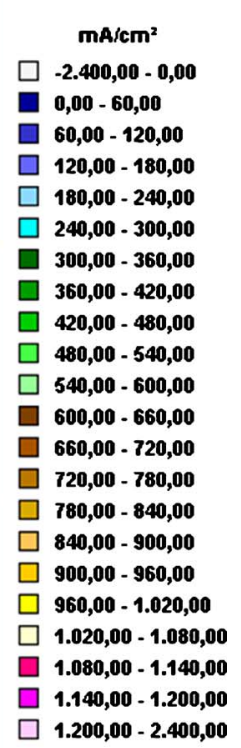

Figure 8. (Color online) Mapping of current density distributions during the evolution of a membrane leakage (absolute values of current density plotted). the membrane and a pronounced temperature gradient for different locations. As a consequence, the membrane will experience mechanical strain due to local dimensional changes which has been reported before. ${ }^{30,31}$ Even though the membrane could be operated stably for some time after the incidence, the premature damage led to a thinning of the membrane which exacerbates the heterogeneous distribution, leading to the future fissures and tearing of the membrane near the edges of the cell holder.

Figure 8 shows a map of current density distribution of the cell at about $48 \mathrm{~h}$ after the accidental pressure drop. The color coding represents a range from negative current densities to current densities

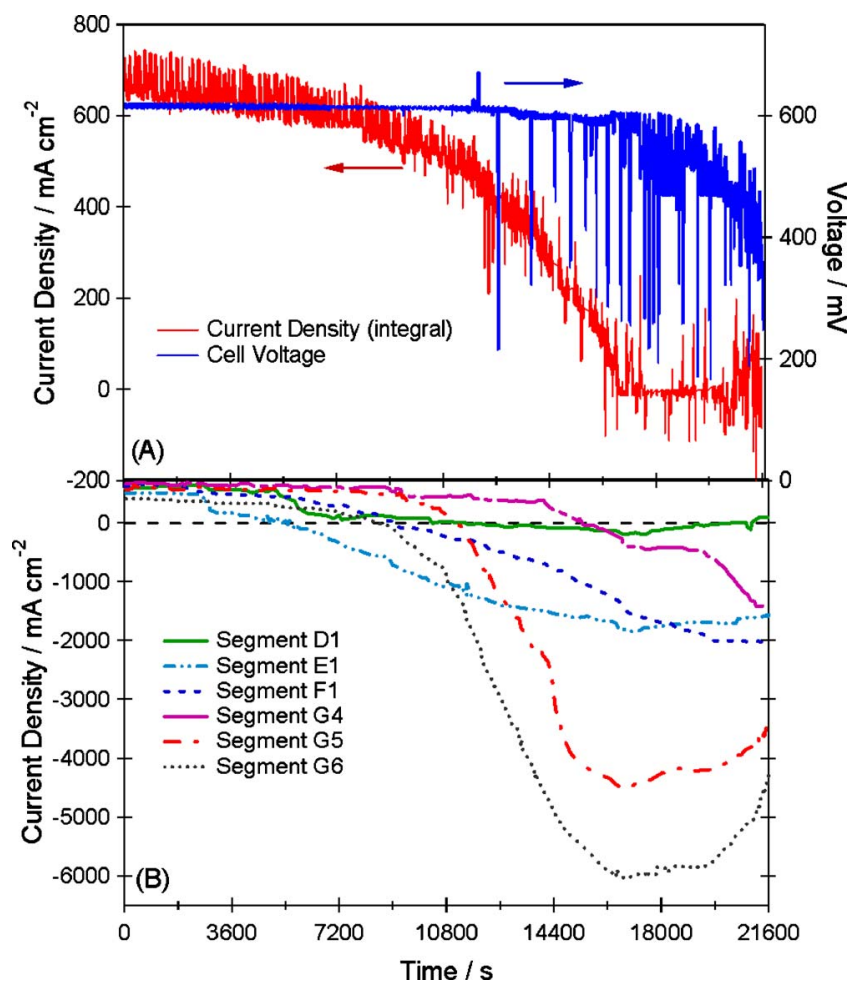

Figure 9. (Color online) Plots of segmented and global current density distribution and open circuit voltage vs time: (a) Global and open circuit voltage and (b) selected segments of the cells (E1, G6, F1, D1, G5 and G4). larger than $1 \mathrm{~A} \mathrm{~cm}^{-2}$. Negative currents have been observed in areas of membrane pinholes. It is observed that a negative current density first appeared in the area of segment (E1) (the value of negative current is indicated by the legend). This indicates that a leakage of the membrane started in the area of segment E1 at the edge of the cell. Since the appearance of negative currents is characteristic for membrane pinholes, the evolution of the cell malfunction can be monitored. After some time, another leakage appeared in G6, which is also near the border of the flow field. Then, more and more leakages (G6, F1, D1, G5, and G4) appear, which were all located in the boundary of the flow field and gasket. Furthermore, the leakage affects the neighboring regions, spreads to the middle of the membrane, and affects an increasing part of the active area. Finally, the membrane is totally destroyed, which is seen by simultaneous strong current and voltage decrease. From the analysis of the images, it is clear that the current drop in the third image in segment E1 is larger compared to the fluctuations under normal operation, even in the presence of flooding. As a consequence, such occurrence can be used as an indication of an imminent failure and could be used for initiation countermeasures or a safe shutdown of the fuel cell system.

Figure 9 shows the plots of global cell current density and voltage versus time as well as locally resolved currents for selected segments for the same incident of the cell at $120^{\circ} \mathrm{C}$.

From the plots of global current versus time (Fig. 8a), it is observed that at potentiostatic operation conditions, the global current density gradually decreases to zero after 4:40 h. In general, fluctuations in the current are evident, which are associated with the high temperature operation of the cell. After the current density, drops to zero, the controlled voltage starts to decrease, indicating the complete failure of the cell function. In Fig. 6b, the time dependences of segments E1, G6, F1, D1, G5, and G4 during the same period are displayed. For clarity, the strong fluctuations of the segment currents have been removed and the lines of the graph indicate only the main time-dependent current trend. It was found that the current density of the segments decreased from positive values to zero and then exhibited large negative current values. The negative current densities can reach exceptionally high values in some segments $\left(-6000 \mathrm{~mA} / \mathrm{cm}^{2}\right)$, in particular G4 and G5 segments. In accordance with the current density mapping, a negative current density first appeared in segment E1, followed by G6, F1, D1, G5, and G4 in this sequence. As described, the observations here are interpreted as indicative of a spreading membrane leakage: the leakage first started at the E1 segment and then another leakage appeared in G6 following the appearance of negative currents (comparing to crossing of 


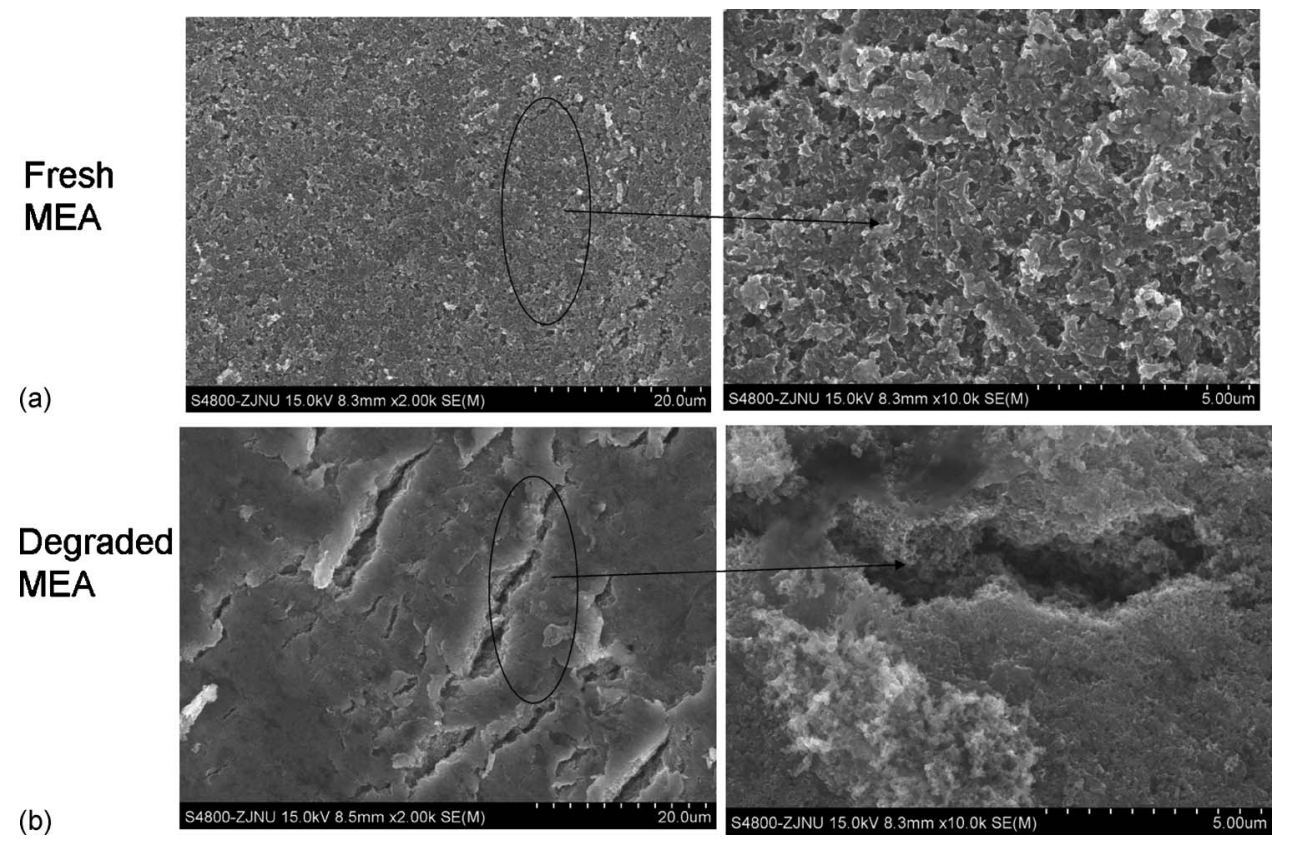

Figure 10. Surface morphologies of the cathode electrode: (a) initial MEA and (b) the one after membrane failure. the zero line). Furthermore, leakages in F1, D1, G5, and G4 segments gradually appeared, which were all located at the boundary of the flow field and gasket. After the first hole forms, the heterogeneous current density and temperature distribution are increased and lead to severe mechanical strain. Finally, the cell voltage decreases gradually even under potentiostatic conditions due to massive gas crossover and mixed potential formation on both sides.

SEM characterization of the ruptured MEA.- The fuel cell MEA was disassembled to explore the degradation mechanism by SEM Figure 10 compares the morphology of the fresh and the failed MEA with the images of the cathode electrode of the unused MEA shown at the top and the degraded cathode electrode displayed at the bottom of the figure. It is evident that the unused MEA possesses uniformly dispersed $\mathrm{Pt} / \mathrm{C}$ catalysts. ${ }^{32}$ In contrast, the degraded MEA shows aggregated catalysts in the layer. More pronounced is the appearance of cracks in most regions of the catalyst layer of the failed MEA. Considering the malfunction of the fuel cell, it is assumed that these structural changes may also be associated with the mechanical strain caused by pressure drop and temperature change during the operation.

\section{Conclusions}

Current density distribution measurements are reported with segmented printed circuit boards introduced both at the anode as well as at the cathode side to prove the equivalence of the distributions at both sides. Even though measurement resistances are introduced into the electrical circuit by the measuring device, the difference of current density distributions is minimal, which indicates that the coupling of both electrodes through the membrane is strong and lateral equalizing currents are not important under these conditions.

The signature of membrane pinholes in the current density distribution is determined for open circuit conditions and under load. It is clear that the current density measurements allow detecting leakages in the cell. Especially at open circuit conditions, large areas operating in the electrolysis mode as well as areas near the pinhole operating in the fuel cell mode coexist under stationary conditions. Under load, the current density distribution is reversed, with large negative currents present in the neighborhood of the pinhole. This effect is explained by the dominance of hydrogen oxidation rates over the oxygen reduction currents at the cathode side. As a consequence, the reaction at the anode side (negative electrode) is also reversed.
This pronounced pinhole signature can be used for monitoring the time development of the failure of fuel cells, which is especially important for high temperature PEMFC. Through the in situ study of the evolution of current density distributions, an evolving membrane leakage is identified. The occurrence of a test stand error is identified as the probable inducing incident for the membrane failure. A variation in local water content and temperature gradient resulted in mechanical strain and stress. The degraded electrodes are characterized by SEM. It is found that the electrodes show cracks in the catalyst layer, which are also the result of mechanical stress.

The application of segmented cells helps to find the causes and mechanisms of cell degradation and their influence on long-term performance can be evaluated. With this knowledge, a catastrophic failure of the whole stack may be avoided by implementing appropriate control systems. Safety and reliability of stack operation can be improved.

\section{Acknowledgments}

The authors gratefully acknowledge the financial support from German Aerospace Center. The authors sincerely thank Heinz Sander for his kind help for the experiment, discussion, and revision of the paper. The authors also thank Renate Hiesgen for manuscript revision. Rui Lin also thanks Alfried Krupp von Bohlen und Halbach-Stiftung association support, the doctoral program of higher education (no. 1700279016), program for young excellent talents in Tongji University (no. 2006KJ022), and National Natural Science Foundation (no. 20703031).

German Aerospace Center assisted in meeting the publication costs of this article.

\section{References}

1. C. Chen and T. F. Fuller, J. Electrochem. Soc., 156, B1218 (2009).

2. C. Chen and T. F. Fuller, Polym. Degrad. Stab., 94, 1436 (2009).

3. M. Kitazawa, A. Y Nosaka, and Y Nosaka, J. Appl. Electrochem., 38, 491 (2008).

4. N. Ramaswamy, N. Hakim, and S. Mukerjee, Electrochim. Acta, 53, 3279 (2008)

5. J. Y. Shim, S. Tsushima, and S. Hirai, J. Electrochem. Soc., 156, B690 (2009).

6. F. M. Collette, C. Lorentz, G. Gebel, and F. Thominette, J. Membr. Sci., 330, 21 (2009).

7. V. A. Sethuraman, J. W. Weidner, A. T. Haug, and L. V. Protsailo, J. Electrochem Soc., 155, B119 (2008)

8. S. Hommura, K. Kawahara, T. Shimohira, and Y. Teraoka, J. Electrochem. Soc., 155, A29 (2008).

9. L. Merlo, A. Ghielmi, L. Cirillo, M. Gebert, and V. Arcella, J. Power Sources, 171, 140 (2007).

10. C. Chen, G. Levitin, D. W. Hess, and T. F. Fuller, J. Power Sources, 169, 288 
(2007).

11. V. O. Mittal, H. R. Kunz, and J. M. Fenton, J. Electrochem. Soc., 154, B652 (2007).

12. S. Kundu, L. C. Simon, and M. W. Fowler, Polym. Degrad. Stab., 93, 214 (2008).

13. V. O. Mittal, H. R. Kunz, and J. M. Fenton, Electrochem. Solid-State Lett., 9, A299 (2006).

14. A. Panchenko, H. Dilger, J. Kerres, M. Hein, A. Ullrich, T. Kaz, and E. Roduner, Phys. Chem. Chem. Phys., 6, 2891 (2004).

15. A. Panchenko, H. Dilger, E. Moller, T. Sixt, and E. Roduner, J. Power Sources, 127, 325 (2004).

16. J. L. Qiao, M. Saito, K. Hayamizu, and T. Okada, J. Electrochem. Soc., 153, A967 (2006).

17. D. A. Schiraldi, Polym. Rev., 46, 315 (2006)

18. A. Z. Weber, J. Electrochem. Soc., 155, B521 (2008).

19. S. J. C. Cleghorn, C. R. Derouin, M. S. Wilson, and S. Gottesfeld, J. Appl. Electrochem., 28, 663 (1998).

20. J. Stumper, S. A. Campbell, D. P. Wilkinson, M. C. Johnson, and M. Davis, Electrochim. Acta, 43, 3773 (1998)

21. C. Wieser, A. Helmbold, and E. Gulzow, J. Appl. Electrochem., 30, 803 (2000).
22. N. Rajalakshmi, M. Raja, and K. S. Dhathathreyan, J. Power Sources, 112, 331 (2002).

23. R. Eckl, R. Grinzinger, and W. Lehnert, J. Power Sources, 154, 171 (2006).

24. P. C. Ghosh, T. Wuster, H. Dohle, N. Kimiaie, J. Mergel, and D. Stolten, J. Power Sources, 154, 184 (2006).

25. D. G. Strickland, S. Litster, and J. G. Santiago, J. Power Sources, 174, 272 (2007).

26. M. Schulze, E. Gulzow, S. Schonbauer, T. Knori, and R. Reissner, J. Power Sources, 173, 19 (2007).

27. M. Schulze, E. Gülzow, K. A. Friedrich, P. Metzger, and G. Schiller, ECS Trans., 5(1), 49 (2007).

28. Y. G. Yoon, W. Y. Lee, T. H. Yang, G. G. Park, and C. S. Kim, J. Power Sources, 118, 193 (2003).

29. T. Knori and M. Schulze, J. Power Sources, 193, 308 (2009).

30. N. Yousfi-Steiner, P. Mocoteguy, D. Candusso, D. Hissel, A. Hernandez, and A. Aslanides, J. Power Sources, 183, 260 (2008).

31. H. Li, Y. H. Tang, Z. W. Wang, Z. Shi, S. H. Wu, D. T. Song, J. L. Zhang, K. Fatih, J. J. Zhang, H. J. Wang, et al, J. Power Sources, 178, 103 (2008).

32. R. Lin, B. Li, Y. P. Hou, and J. M. Ma, Int. J. Hydrogen Energy, 34, 2369 (2009). 